\title{
Saberes etozoológicos de los pastores andinos: su importancia para la conservación y manejo de la vicuña (Vicugna vicugna)
}

\author{
ANA C. WAWRZYK \\ Grupo de investigación VICAM: Vicuñas, camélidos y ambiente; Departamento de Ciencias Sociales; Universidad Nacional de Luján
}

\begin{abstract}
Resumen. El interés sobre los saberes etnobiológicos de los pueblos originarios se ha incrementado en las últimas décadas. La evidencia científica señala que dichos conocimientos pueden contribuir a la conservación de la biodiversidad, la preservación de áreas protegidas y el uso sustentable de los recursos naturales. Los pobladores andinos poseen conocimientos y saberes sobre el uso de los recursos naturales basados en sus propias prácticas y vivencias vinculadas con la actividad del pastoreo. En tiempos en los que los recursos naturales y la fauna silvestre se encuentran bajo una presión sin precedentes es fundamental analizar cómo las relaciones humanas con la vida silvestre pueden influir en los planes de manejo y conservación. A partir del uso de diversas técnicas de investigación social, en especial las entrevistas en profundidad realizadas a pastores de cuatro localidades de la puna Jujeña: Cieneguillas, Suripujio, Susques y Lagunillas del Farallón, se indagó sobre los conocimientos etnozoológicos sobre las vicuñas silvestres que habitan la región. En general se observó que los pastores de las cuatro localidades reconocen que las vicuñas conforman grupos familiares y grupos de machos solitarios, aunque debido a la ausencia de dimorfismo sexual resulta difícil poder distinguir si se trata de machos o hembras y cuál es la proporción de cada uno de ellos. Asimismo, señalan que las vicuñas prefieren las mejores pasturas y permanecen en sitios con disponibilidad de agua. En algunos casos los conocimientos de los pobladores locales en relación a la especie resultan incompletos o incluso contradicen al conocimiento científico. Indagar sobre los saberes ecológicos tradicionales acerca de las vicuñas silvestres puede contribuir al diseño de planes de manejo y conservación que permitan adaptarse a la realidad local revalorizando la cosmovisión andina.
\end{abstract}

[Palabras clave: Altiplano, percepción, pobladores local, conocimientos ecológicos tradicionales]

\begin{abstract}
Aвstract. Ethnoecological knowledge of pastoral Andeans: its importance for the conservation and management of the vicuña (Vicugna vicugna): The interest regarding the ethnobiological knowledge of indigenous peoples has increased in the last decades. Evidence indicates that such knowledge contributes to biodiversity conservation, to the preservation of protected areas and to the sustainable use of natural resources. Andean people have knowledge and skills about the use of natural resources based on their own practices and experiences, related mainly with the grazing activity. Because natural resources and wildlife are under an unprecedented pressure from human activity, it is essential to analyze how the human relationship with wildlife influences management and conservation plans. We performed interviews in the Puna jujeña, in the towns of Cieneguillas, Suripujio, Susques y Lagunillas del Farallón. I conducted inquiries regarding the biological and behavioral knowledge of the wild vicuña inhabiting the region. In general, pastorals of the four localities recognize that the vicuña are organized in family groups and solitary males groups, but due to the absence of sexual dimorphism, it is difficult to distinguish between male and female and their relative proportions. They also note that the vicuña prefer the best pastures and stay in places with water availability. In some cases, local people knowledge in relation to the species is incomplete or disagree with scientific data. Understanding the traditional ecological knowledge of the wild vicuña contributes to the design of conservation and management plans, which can be adapted to local realities by revaluing the Andean worldview.
\end{abstract}

[Keywords: highlands management and conservation, local people, perception, traditional ecological knowledge]

\section{INTRODUCCIÓN}

Los animales silvestres han ocupado un lugar preponderante en el imaginario y en las representaciones de los diferentes pueblos alrededor del mundo. A la vez, desempeñan un papel importante en los sistemas socioeconómicos, culturales, estéticos, recreativos y espirituales del ser humano (Robinson \& Redford 1997; Descola 2004; Chardonnet et al. 2002; Morckrin et al. 2005). La fauna siempre ha constituido una parte esencial y significativa de la realidad y de la cotidianeidad de los grupos humanos. Los vínculos cognitivos, emocionales y comportamentales que se establecen han jugado un rol clave en las diferentes idiosincrasias y relaciones con los componentes del universo de diversos grupos culturales (González 2001). En este sentido, la importancia que un determinado animal tiene para cierta sociedad depende de cómo se concibe y representa dentro de este grupo social. Su simbolismo y valor se vincula con 
las percepciones, conocimientos y significados de cada individuo o la comunidad en relación a esa especie (Vargas Clavijo 2009).

Para comprender la complejidad de las relaciones de las poblaciones humanas con la fauna deben considerarse tres componentes básicos: el cognitivo (concepciones y saberes), el afectivo (relaciones afectivas y emocionales) y el conductual (actitudes hacia las especies) (Piaget 1969; Descola 1986). Mediante la etnozoología se intenta integrar estos tres pilares para comprender de manera holística las relaciones de las poblaciones con la fauna. La mayoría de las actitudes hacia los animales son consecuencia de los valores adquiridos, de la percepción, del conocimiento sobre la especie y de las relaciones sociedad-especie. Por lo tanto, el estudio de los valores, las actitudes, las normas, las motivaciones y satisfacciones son cruciales e imprescindibles al momento de diseñar planes conservación y uso de las especies (Kellert 1996).

Todas las especies animales tienen un papel clave, propio y único en los ecosistemas donde habitan. Sin embargo, no es este el valor principal sobre el que se basa el éxito de los proyectos de conservación, sino que está más determinado por la subjetividad en relación con la especie. Las razones de preferencias hacia determinadas especies o grupo de taxones varían de forma significativa entre distintos grupos humanos e involucran una innumerable cantidad de factores evolutivos, genéticos, psisociales y culturales (Kellert \& Wilson 1993). Turbay (2002) señala que la percepción, las relaciones y las actitudes que los seres humanos tienen sobre los animales dependen de factores ecológicos, geográficos, históricos, económicos, psicológicos, filosóficos, sociales y culturales, propios de las circunstancias temporales y espaciales de cada grupo social.

Los animales están arraigados en los múltiples esquemas simbólicos, espirituales y culturales de nuestras identidades a través de mitos, leyendas, sueños, fantasías, folclore y arte (Urton 1985; Lawrence 1993). Los saberes etnobiológicos que los pastores andinos han desarrollado y transmitido entre generaciones sobre el ambiente, los animales, las plantas, el clima, los astros, los seres sobrenaturales, conforman un cúmulo de experiencias que contribuyen a la generación de conocimientos e influyen sobre el modo de percibir y de relacionarse con el medio. Existe un consenso creciente acerca de que las prácticas tradicionales constituyen una rica y valiosa fuente de información sobre los múltiples componentes ambientales, como así también sobre la construcción, el funcionamiento y la conservación (Berkes et al. 2000; Turner 2000; Pieroni 2001). Es por ello que resulta importante estudiar los saberes etnozoológicos de una especie tan emblemática para la cosmovisión de los pueblos andinos como es la vicuña para diseñar políticas públicas que tengan en cuenta estos conocimientos para favorecer su conservación.

\section{Área de estudio}

La investigación se llevó a cabo en cuatro comunidades de la puna jujeña (Argentina) ubicadas a más de 3000 m.s.n.m.: Cieneguillas, Suripujio, Lagunillas del Farallón y Susques. La principal actividad económica es la ganadería de altura. Las localidades fueron seleccionadas según diversas variables ambientales y sociales, entre ellas: la densidad de vicuñas en el área, la organización social de la comunidad y la experiencia en el manejo de la especie, entre otras (Tabla 1).

La localidad de Cieneguillas $\left(22^{\circ} 06^{\prime} 02^{\prime \prime} \mathrm{S}\right.$ y $65^{\circ} 52^{\prime} 01^{\prime \prime}$ O) se encuentra en el Departamento de Santa Catalina, $37 \mathrm{~km}$ al oeste de la localidad de La Quiaca, a una altura promedio de 3672 m.s.n.m. (Braun Wilke 2001). Cieneguillas es un pueblo de 270 habitantes (INDEC 2001), y se diferencia de otras localidades de la puna porque allí la propiedad de la tierra es privada y en la mayoría de los casos las parcelas se encuentran alambradas para limitar el acceso. La principal actividad económica es la cría de ovinos para la producción de carne y lana. Además, se crían camélidos domésticos (llamas) para la producción de fibra, aunque en los últimos años ha crecido la producción de carne. Otra caracteristica que diferencia la localidad de Cieneguillas del resto de las comunidades de la puna es la elevada densidad de vicuñas en el área (densidad media 12 vicuñas / $\mathrm{Km} 2$ ), una de las mayores de la provincia de Jujuy (Arzamendia \& Vilá 2006). Como reconocimiento

Tabla 1. Características de las cuatro comunidades rurales estudiadas en la puna jujeña.

Table 1. Characteristics of the four studied rural communities in the Puna Jujeña.

\begin{tabular}{|c|c|c|c|c|}
\hline Localidad & $\begin{array}{l}\text { Densidad } \\
\text { de V } / \mathrm{Km}^{2} \\
\text { Censo } \\
\text { Propio }\end{array}$ & $\begin{array}{l}\text { Densidad } \\
\text { Censo } \\
\text { Nacional }\end{array}$ & $\begin{array}{l}\text { Experiencia } \\
\text { de Manejo }\end{array}$ & $\begin{array}{l}\text { Tenencia } \\
\text { de la } \\
\text { tierra }\end{array}$ \\
\hline Cieneguillas & $10-12$ & Muy alta & $\mathrm{Si}$ & Priv \\
\hline Surip & $3.9-4.9$ & Alta & No & Comunal \\
\hline $\begin{array}{l}\text { Lagunillas } \\
\text { del Farallón }\end{array}$ & $3.2-4.5$ & Media & No & $\begin{array}{c}\text { Comunal } \\
\text { y } \\
\text { privadas }\end{array}$ \\
\hline Susques & 1.5- 4 & Baja & No & Comunal \\
\hline
\end{tabular}


a la actitud conservacionista adoptada durante años, en el 2002 la localidad de Cieneguillas fue declarada "Pueblo Protector de las Vicuñas" por la Honorable Cámara de Diputados de la Nación. Esta localidad en particular reviste interés para esta investigación ya que es la primera comunidad (de las cuatro estudiadas) que ha capturado y esquilado vicuñas silvestres.

La localidad de Lagunillas del Farallón (22²3'60" S y 66 $6^{\circ} 0^{\prime \prime} \mathrm{O}$, Departamento de Rinconada, en la cuenca del Río Grande de San Juan), está conformada por 40 familias y 350 habitantes (180 hombres y 170 mujeres). Está ubicada a 4165 m.s.n.m. (Braun Wilke 2001). Los medios de vida se circunscriben a la ganadería de subsistencia de llamas, ovejas y cabras y al empleo público (Wawrzyk \& Vilá 2013). En el área de influencia de la localidad de Lagunillas la densidad media de vicuñas es de 4.5 vicuñas $/ \mathrm{km}^{2}$, con áreas de alta concentración de animales que pueden alcanzar las 8 vicuñas $/ \mathrm{km}^{2}$ (Wawrzyk et al. 2009).

La localidad de Suripujio $\left(22^{\circ} 10^{\prime} 0^{\prime \prime}\right.$ S y $\left.65^{\circ} 2^{\prime} 0^{\prime \prime} \mathrm{O}\right)$ está ubicada a $34 \mathrm{~km}$ de La Quiaca, en el Departamento de Yavi, sobre una planicie elevada a 3739 m.s.n.m. (Braun Wilke 2001). Es una población rural dispersa, con una población en descenso constante, entre el 2008 y el 2010 la cantidad de familias en la comunidad decreció de 32 a 24 (comunicación personal, pobladores del lugar) (Quiroga Mendiola \& Ramisch 2010). La economía es de subsistencia, basada en la cría de ovinos y camélidos domésticos, complementada con la agricultura a pequeña escala de tubérculos, cereales y hortalizas. En las áreas de pastoreo las vicuñas comparten los recursos con los animales domésticos (llamas, ovejas, burros y vacas), que alcanzan densidades de $4-5$ vicuñas $/ \mathrm{km}^{2}$ (censo realizado por investigadores del grupo VICAM, 2008 (Rojo et al. 2012).

La localidad de Susques $\left(23^{\circ} 25^{\prime} 0^{\prime \prime} \mathrm{S}\right.$ y $66^{\circ} 28^{\prime} 60^{\prime \prime}$ O) se encuentra al sudoeste de la provincia, en la región conocida como Puna seca o de Atacama, a 3675 m.s.n.m. (Braun Wilke 2001). Susques dista 198 $\mathrm{km}$ de San Salvador de Jujuy y $124 \mathrm{~km}$ de la frontera con Chile. La economía se desarrolla en torno a la ganadería de camélidos domésticos, ovejas y cabras, a la que se suma la actividad minera. A diferencia del resto de las comunidades seleccionadas, en las proximidades del pueblo de Susques la densidad de vicuñas es baja. Sin embargo, en el área de influencia de la comunidad se encuentra la Reserva Provincial de Olaroz Cauchari, donde la densidad de vicuñas aumenta significativamente hasta 5 vicuñas $/ \mathrm{km}^{2}$ (censo realizado por investigadores del grupo VICAM 2008 inédito).

\section{MATERIALES y MÉTODOS}

Con el fin de descubrir cómo el hombre común construye su mundo de experiencia y cómo lo ordena, categoriza y clasifica, los antropólogos cognitivos han llegado a la etnociencia (Sturtevant
1968). La etnociencia asocia al enfoque émico (Pike 1954), "la visión desde dentro" o "la visión del actor" que conoce, comprende y comunica los fenómenos de su propia realidad tal como él los percibe (Frake 1969). La etnozoología ordena y codifica los conocimientos empíricos acumulados por la cultura tradicional acerca del reino animal. Se trata de una ciencia interdisciplinaria cuyo cuerpo metodológico se construye a partir del aporte de los métodos de las ciencias naturales y humanas. Este trabajo combinó técnicas y metodologías que siguieron los lineamientos del método etnográfico (Emerson et al. 1995; Guber 2001; Santos Rodríguez 2009). Se emplearon técnicas de observación participante, de registro de historias orales y de entrevistas semiestructuradas (ver Anexo 1 en Información Suplementaria). En un período de 3 años (2006-2008) se entrevistaron un total de 59 hombres y mujeres de entre 20 y 74 años (Cieneguillas 11, Susques 11, Lagunillas del Farallón 18 y Suripujio 19). Se trabajó sobre los conocimientos etológicos de la especie, enfatizando el análisis en su organización social y comportamiento.

\section{Resultados}

\section{Organización social de las vicuñas}

El primer aspecto que aparece en las encuestas se basa en comentarios acerca del tamaño de los grupos. En el 38\% de los casos mencionaron la cantidad de animales sin hacer distinción entre sexo o edad de los individuos. El 38\% de los entrevistados reconoció que las vicuñas forman grupos familiares de entre 6 y 19 animales. Sin embargo, en Lagunillas del Farallón, más de 30\% de los encuestados señaló que las vicuñas -con territorios en áreas próximas a las zonas urbanas- conforman grupos pequeños de entre 2 y 5 animales. Los grupos de vicuñas que habitan zonas altas de pastoreo son más numerosos. En las cuatro localidades, los entrevistados coincidieron en que en la actualidad las tropas de vicuñas son más numerosas que dos o tres décadas atrás. Por otro lado, $17 \%$ de los entrevistados mencionaron haber visto grupos de vicuñas de más de 40 animales, y reconocieron que se trata de grupos de machos solteros (Figura 1).

En cuanto a la conformación de los grupos, se identificaron cinco categorías de análisis: grupos de machos solteros, grupos familiares, individuos solitarios, individuos mezclados (es decir, no han identificado el tipo de organización social), y grupos no identificados (los que demostraron no poder identificar si se trata de machos o hembras). La mayoría de los entrevistados (53\%) mencionó 


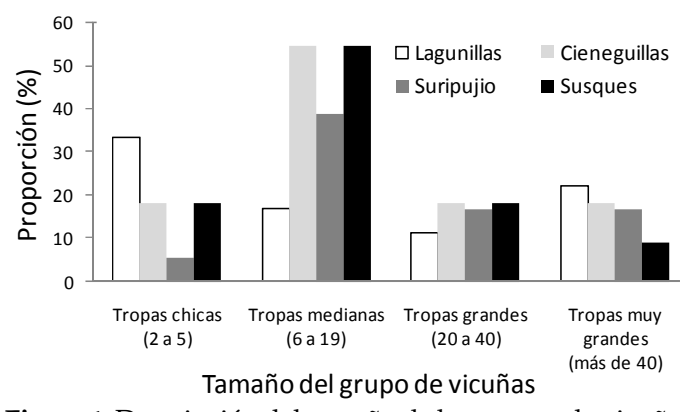

Figura 1. Descripción del tamaño de los grupos de vicuñas en cuatro localidades de la puna jujeña.

Figure 1. Description about the size of vicuña's groups in each of four sites in the Puna Jujeña.

dos formas básicas de organización social de la especie: grupos familiares y grupos de machos solteros, señalando que es posible distinguir estos grupos debido a su tamaño y comportamiento individual.

Algunos entrevistados señalaron que debido a las similitudes morfológicas entre ambos sexos, resulta imposible distinguir si se trata de machos o hembras. Además, algunos afirmaron que los grupos familiares se componen de hembras y machos indistintamente (Figura 2).

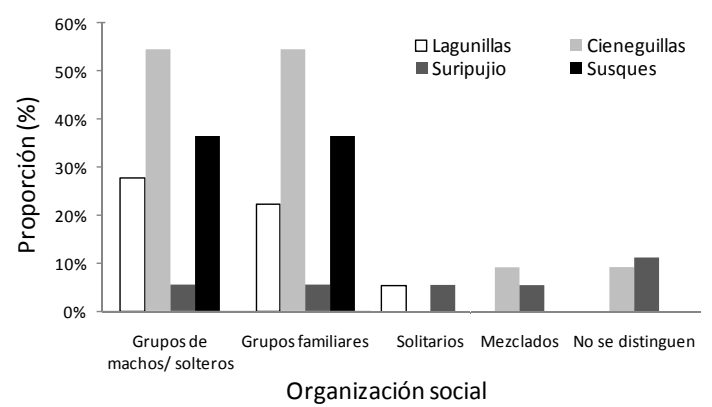

Figura 2. Descripción acerca de la organización social de la vicuña en cada una de cuatro localidades de la puna jujeña.

Figure 2. Description about the social organization of the vicuña in each of four sites in the Puna Jujeña.

\section{Comportamiento de las vicuñas}

Los conocimientos de los encuestados en relación al comportamiento y hábitos de la especie se agruparon en distintas categorías de análisis que abarcan: el comportamiento en relación a la organización social; el carácter de la espacie, y el comportamiento en función de los sitios de pastoreo.

El comportamiento en relación a la organización social. El $44 \%$ de los encuestados de Lagunillas del Farallón hizo referencia a algún tipo de comportamiento relacionado con la organización social de la especie, mientras que en Suripujio sólo el $21 \%$ de los entrevistados hizo algún tipo de mención al respecto(Tabla 2). El comportamiento señalado con mayor frecuencia fue la pelea entre machos $(20 \%)$, generalmente referida a las expulsión de las crías en edad juvenil, a la defensa del grupo familiar y a conseguir o "robar" hembras para conformar un nuevo grupo familiar. En ningún caso los entrevistados hicieron referencia al comportamiento no agresivo relacionado con la territorialidad, de vigilancia del área de hembras.

Tabla 2. Respuestas afirmativas sobre el conocimiento del comportamiento de las vicunas.

Table 2. Affirmative answers about behavioral knowledge of the vicuña.

\begin{tabular}{lccc}
\hline Localidad & $\begin{array}{l}\text { Respuestas } \\
\text { afirmativas }\end{array}$ & Total entrevistados & $\%$ \\
\hline Lagunillas & 8 & 18 & 44 \\
Cieneguillas & 7 & 11 & 39 \\
Suripujio & 5 & 19 & 21 \\
Susques & 6 & 11 & 35 \\
Total & 26 & 59 & 44 \\
\hline
\end{tabular}

El 18\% del total de entrevistados identificó que los grupos familiares están constituidos por un único macho quien pastorea junto a las hembras y sus crías. Asimismo, 15\% hizo mención a la conformación de tropas de machos solteros, mientras que $13 \%$ detalló que estos grupos de solteros se conforman a partir de la expulsión del grupo familiar por parte del "relincho" de las crías en edad juveniles.

A pesar de que en numerosas oportunidades los entrevistados mencionaron la existencia de hembras con sus crías, en ninguno de los casos se describieron comportamientos sobre la relación madre cría, como el amamantamiento o los cuidados parentales. El 18\% de los entrevistados señaló la permanencia de las madres con sus crías y sólo en casos aislados se refirieron al periodo de parición. A pesar de que el comportamiento de alerta en los machos familiares es muy frecuente, sólo 2 entrevistados lo mencionaron.

El análisis por localidad mostró que existen diferencias en las descripciones de los comportamientos de las vicuñas en relación a la organización social de la especie. En Suripujio, por ejemplo, no se registraron relatos referentes a la conformación de los grupos de solteros, mientras que en Cieneguillas, 7 de los 11 entrevistados mencionaron el tema. Del mismo modo, los entrevistados de Lagunillas del Farallón no mencionaron la expulsión de las crías 
en edad juvenil; este relato también se observó en el resto de las localidades (Figura 3).

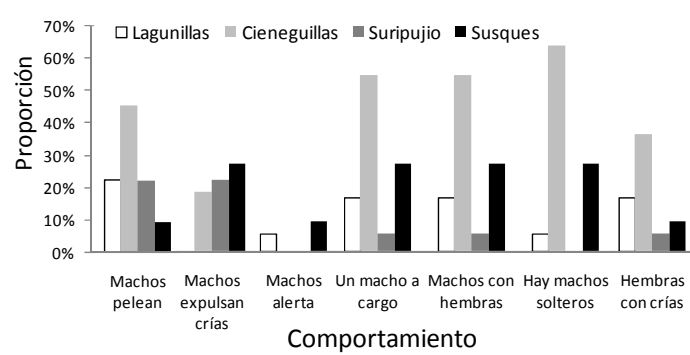

Figura 3. Proporción de afirmaciones en relación al comportamiento de las vicuñas en cuatro localidades de la puna jujeña.

Figure 3. Proportion of affirmations in relation to the vicuña's behaviour in each of four sites of the Puna Jujeña.

El comportamiento en relación al carácter de la especie. El 43\% de los encuestados describió que las vicuñas son "chúcaras" y "ariscas"; esta característica sobresalió en relación al carácter de la especie. Sin embargo, el 26\% señaló que hay vicuñas mansas y tranquilas. Este antagonismo fue explicado por el lugar en donde se encuentran las vicuñas: aquellas que viven en el cerro o en campos abiertos son más "ariscas" que las que pastorean cerca de zonas urbanas o caminos.

Por otro lado, el $44 \%$ de los encuestados de Suripujio afirmó que las vicuñas presentan un comportamiento de huida y escape al ver o escuchar gente, mientras que en Cieneguillas sólo el $9 \%$ hizo mención a este comportamiento, lo que podría indicar que estas poblaciones de vicuñas están más habituadas a la gente, la hacienda y los vehículos. En muchos casos, los entrevistados reconocieron que estos compartimientos se correspondían con las características de una especie silvestre. En contrapartida a la actitud huidiza de las vicuñas, el 30\% de los entrevistados señaló que con el paso de los años, las vicuñas se estaban habituando a la gente y a la hacienda, y remarcó que ya no "disparaban" como antes o que -incluso- se quedaban mirando atentas cuando pasaban a su lado.

\section{Uso del hábitat}

Del análisis de los discursos, $21 \%$ de los encuestados indicó que las vicuñas preferían sitios con disponibilidad de agua y que los movimientos diarios y estacionales estaban determinados por este recurso. Notablemente esta afirmación se profundizó en Suripujio, donde $50 \%$ de los entrevistados mencionaron este comportamiento. La disponibilidad de agua en esta localidad es baja (principalmente en época invernal), por lo que las vicuñas tienden a concentrarse en las vegas o cursos de agua. En cambio, en Lagunillas del Farallón ninguno de los entrevistados mencionó que las vicuñas prefieran lugares con disponibilidad de agua, probablemente porque el recurso permanece disponible la mayor parte del año. Mientras tanto, en Suripujio el déficit hídrico en épocas invernales es mayor, por lo que se debe trasladar la hacienda a las localidades de Burrería o Chalguamayo en busca de agua (Figura 4). Por otro lado, el $19 \%$ de los encuestados afirmó que las vicuñas permanecían en las mejores pasturas. Esta afirmación fue más frecuente en Cieneguillas, donde el 55\% de los entrevistados en 2009 mencionóestecomportamiento(probablemente debido a que la siembra de pasturas para la hacienda es una práctica frecuente en la localidad). Del mismo modo, los pobladores de Suripujio reconocieron que las vicuñas preferían los pastos más blandos y tiernos que reservan para la hacienda (Figura 4).
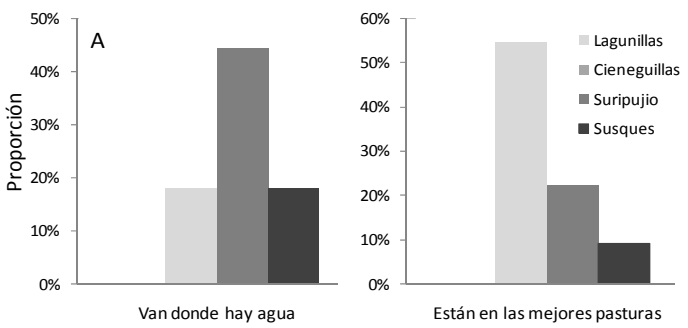

Figura 4. Afirmaciones en relación a la preferencia de las vicuñas por sitios con disponibilidad de agua (A) y mejores pasturas (B).

Figure 4. Affirmation related to the vicuña's preferences for places with water availability (A) and better pastures (B).

\section{DisCUSIÓN}

En tiempos donde los recursos naturales y la fauna silvestre se encuentran bajo una presión sin precedentes es fundamental analizar cómo las relaciones humanas con la vida silvestre pueden influir en los planes de manejo y conservación. Tal como defiende el antropólogo Claude Lévi- Strauss (1962), las especies animales y vegetales son útiles $\mathrm{e}$ interesantes porque primero son conocidas. En este sentido, observamos que, en general, los pobladores delas cuatrolocalidades estudiadas poseen conocimientos etnozoológicos empíricos sobre las vicuñas silvestres los que en algunos casos pueden no corresponderse con conocimientos científicos. 
La organización social de la vicuña incluye los grupos familiares, los grupos de solteros y los individuos solitarios (Koford 1957; Franklin 1974). En general, los pobladores locales mencionan grupos más numerosos que los observados sistemáticamente. Esto sucede tanto para los grupos familiares como para los grupos de machos solteros probablemente porque en una misma área pueden permanecer diversos grupos que los pobladores identifican como una única unidad. De acuerdo con las investigaciones, los grupos familiares están integrados en promedio por un macho dominante, entre tres a cuatro hembras y dos crías (Koford 1957; Frankin 1983; Vilá \& Cassini 1994; Renaudeau d'Arc \& Vilá 1998; Arzamendia 2008). El tamaño de los grupos de machos solteros es muy variable. Estos grupos se caracterizan por su gran movilidad y porque las fisiones y fusiones entre los mismos suelen ser muy comunes (Koford 1957; Franklin 1982). En más de una década de censos continuos en la puna nunca se registraron tropas que superaran los 70 animales. Sin embargo, en las cuatro localidades estudiadas los pastores afirman haber visto grupos que superan el centenar de animales.

La etología de las vicuñas es otro aspecto conocido por los pastores de la puna. Dada la carencia de dimorfismo sexual de la especie, muchos de los entrevistados señalaron las dificultades para diferenciar a los machos de las hembras. Sin embargo, algunos pueden distinguirlos a partir del comportamiento de los individuos. Los relatos se refirieron en su mayoría al comportamiento de los grupos en torno al uso del hábitat y sus hábitos de alimentación y desplazamiento. Asimismo, se observó una clara tendencia a reconocer el comportamiento de los machos, mientras que la información revelada en relación a las hembras y las crías fue escasa.

Los machos son los encargados de mantener el número de animales de su grupo familiar (Vilá 1992) y de evitar que las hembras puedan aparearse con otros machos. Esto lo logran con comportamientos agresivos. Por esta razón, uno de los principales comportamientos identificados por los entrevistados ha sido la pelea entre machos y la expulsión de los machos juveniles de las familias.

El sistema de apareamiento de las vicuñas tiene componentes mixtos de poliginia de defensa de recursos (i.e., un macho defiende un territorio con recursos para atraer hembras,
Emlen \& Oring 1977) y defensa de harén (Franklin 1983; Vilá \& Cassini 1994). Los pobladores locales reconocen que los machos defienden a las hembras y es por ello que permanecen más tiempo alertas y suelen ser más agresivos. En ninguna de las entrevistas se hizo referencia a la defensa de un territorio.

Respecto del comportamiento maternal, las vicuñas, como el resto de los ungulados, son clasificadas como seguidoras y escondedoras (Lent 1974; Vilá 1992). Además, la distancia que mantienen la madre y la cría es fundamental para la defensa antidepredatoria (Lent 1974; Leuthold 1977; Vilá 1992). A pesar de ser comportamientos habituales entre madres y crías, la frecuencia de relatos que han hecho referencia a este vínculo ha sido muy baja. Los relatos señalan que las crías permanecen con las madres la mayor parte del tiempo. En ninguna de las entrevistas se han revelado detalles sobre el alumbramiento o el amamantamiento de las crías. Tampoco se han registrado comportamientos relacionados con las crías, como pueden ser los juegos, las agresiones, etc.

En cuanto al uso del hábitat, los entrevistados identificaron que las vicuñas se mantienen en áreas determinadas, y señalaron que los grupos familiares son más sedentarios en comparación a los grupos solitarios, que suelen ser más móviles. Los patrones de movimiento de la especie dependen principalmente de la disponibilidad de recursos ambientales (Koford 1957; Franklin 1982; Vilá \& Cassini 1994). Los pobladores señalaron que prefieren sitios con disponibilidad de agua; esto se debe a que las vicuñas son bebedoras obligadas (Franklin 1983). Asimismo, señalaron que prefieren sitios con buena calidad de pasturas y que permanecen más tiempo en las vegas y pastizales tiernos. Por otro lado, en algunos relatos se mencionó que la vicuña come todo el tiempo. La baja calidad y cantidad de recursos forrajeros disponibles en el altiplano determina que las vicuñas pasen la mayor parte del tiempo activo buscando alimento. Esta situación es más significativa en las hembras ya que deben gestar y amamantar a sus crías simultáneamente; destinan entre el 70 y el $90 \%$ del tiempo a la alimentación (Menard 1982; Vilá \& Cassini 1994; Arzamendia 2008). Como la mayoría de los ungulados, las vicuñas pasan la mayor parte del tiempo alimentándose (Franklin 1974; Ménard 1982; Vila \& Cassini 1993; Arzamedia \& Vilá 2003), pero a diferencia de otras especies (ovinos, 
caprinos, vacunos, etc.), han desarrollado una serie de adaptaciones al medio que hacen que su pastoreo sea de bajo impacto. Entre las principales adaptaciones podemos señalar el desarrollo de una fosa en la porción facial del hueso maxilar que le permite usar los labios superiores para aprehender el pasto y traccionarlo sin arrancar de raíz gracias a una presión contra los incisivos inferiores. También la presencia de dientes capaces de masticar vegetación abrasiva y el desarrollo de cojines plantales que les permiten habitar sustratos delicados sin dañarlos (Bonacic 1991; Bas \& Bonacic 2003). Aproximadamente un 10\% de los entrevistados señaló que las vicuñas se alimentan de manera permanente cuando los animales suelen tener ciclos circadianos de comportamiento y más de $12 \mathrm{~h}$ de descanso.

La mayoría de los entrevistados indicó que las vicuñas suelen ser animales ariscos, sobre todo aquellos que habitan zonas aisladas con baja carga ganadera y poca o nula presencia de pastores, características típicas de un animal silvestre. Sin embargo, las vicuñas que habitan áreas con presencia de poblaciones humanas o hacienda suelen ser más tranquilas debido a un proceso de habituación. Además, los entrevistados reconocieron diferentes distancias de huida entre ambos grupos y remarcaron que los grupos más aislados ante el menor disturbio huyen rápidamente por cientos de metros, mientras que en los grupos más habituados a la presencia del hombre las distancias de huida son mucho menores y que permanecen en su territorio en alerta.

Tal como sucede en la mayoría de los saberes tradicionales de los pueblos originarios, la transmisión de conocimientos sobre el ambiente es mediante el lenguaje oral intergeneracional. Sin embargo, los cambios en los estilos de vida producidos por la globalización y el acceso a nuevas formas de conocimiento hacen que estos saberes corran el riesgo de ser olvidados. Muchos de los encuestados, en particular los más jóvenes, poseen un bagaje de conocimientos ancestrales menos diverso que sus antepasados, situación que pone en riesgo a los saberes tradicionales de los pueblos andinos. Por ello es que se requieren estudios etnobiológicos en profundidad que permitan comprender la cosmovisión de los pueblos andinos en una realidad cambiante, donde la globalización y el capital condicionan las prácticas culturales.

Cotejar los saberes etnozoológicos de los pastores andinos desde una visión émica con los conocimientos científicos actuales pude generar situaciones de tensión al momento del diseño de políticas públicas de manejo y uso sustentable de las vicuñas. En general, cuando se presentan situaciones de conflicto entre el ganado doméstico y las vicuñas, fundamentalmente por la disponibilidad de recursos (pasturas y agua), los pastores suelen tener una visión más negativa y percibir una densidad de vicuñas más elevada como estrategia para implementar los planes de manejo de la especie. Sin embargo, el manejo de vicuñas requiere densidades mínimas que son definidas por los investigadores en función de las características ambientales y poblacionales de la especie (Arzamendia \& Vilá 2006). Para poder desdibujar estas diferencias y llegar a acuerdos consensuados con las comunidades es esencial trabajar bajo un marco de participación en el que los pobladores locales sean los propios protagonistas del cambio.

Agradecimientos: A los pobladores de Lagunillas del Farallón, Susques, Suripujio y Cieneguillas por la hospitalidad, la buena predisposición y el tiempo dedicado y por permitirnos ser parte de sus tareas cotidianas viviendo experiencias inolvidables. A B. Vilá por la lectura y los comentarios sobre este manuscrito. A los miembros de VICAM por el apoyo recibido durante la investigación, en especial a Y. Arzamendia, J. Baldo, H. Yacobaccio, V. Rojo y M. Borgnia, con quienes compartimos el trabajo de campo. A C. Campos quien hizo posible que las investigaciones etnobiológicos sean publicadas en esta sección especial. A H. Lamas por sus consejos y saberes sobre las comunidades andinas.Este trabajo se realizó con el subsidio de la ANPCyT, PICT 0306-2010.

\section{BIBLIOGRAFÍA}

ARZAmEndia, Y \& B VILÁ. 2006. Estudios etoecológicos de vicuñas en el marco de un plan de manejo sustentable: Cieneguillas, Jujuy. Cap. 4. Pp. 37-50 en: Vila, B (ed.). Investigación, Conservación y Manejo de Vicuñas. Talleres gráficos Leograf. Buenos Aires, Argentina.

ArZAMENDia, Y. 2008. Estudios etoecológicos de vicuñas (Vicugna vicugna) en relación a su manejo sostenido en silvestría, en la reserva de la biosfera laguna de Pozuelos (Jujuy, Argentina). Tesis Doctoral. Universidad Nacional de Córdoba.

BAird, TD; PW Leslie \& JT Mc CABE. 2009. The effect of wildlife conservation on local perceptions of risk and behavioral response. Hum. Ecol., 37:463-474.

BAS, F \& C Bonacic. 2003. Adaptive strategies of South American Camelids. Pp. 21-33 en: Mannetje, LT; L Ramirez-Aviles; C Sandoval-Castro \& J Kuvera (eds.). VI International Symposium on the nutrition of herbivores.. Universidad Autónoma de Yucatán, Mérida.

Berkes, F; J Colding \& C Folke. 2000. Rediscovery of traditional ecological knowledge as adaptive management. Ecol. Appl., 10:1251-1262.

BONACIC, C. 1991. Características biológicas y productivas de los Camélidos Sudamericanos. Avances en Ciencias Veterinarias, 6:3-15. 
BRAUN WILKE, RH. 2001. Carta de aptitud ambiental de la provincia de Jujuy. Departamento de suelos y ecología de la Facultad de Ciencias Agrarias, Universidad Nacional de Jujuy.

Chardonnet, PH; B Des Clers; J Fischer; R Gerhold; F JORI; ET AL. 2002. The value of life. Rev. Sci. Tech. Off. Int. Epiz., 21(1):15-51.

Descola, P. 1986. La nature domestique: Symbolisme et praxis dans l'écologie des Achuar. Maison des Sciences de L'Homme. París.

Descola, P. 2004. Las cosmologías indígenas de la Amazonía. Pp. 25-35 en: Surrallés, A \& P Garcia (eds.). Tierra adentros, territorio indígena y percepción del entorno. Grupo Internacional de Trabajo Sobre Asuntos Indígenas IWGIA. Tarea Gráfica Educativa. Lima, Perú.

EMERSON, RM;RIFrETz \& LLSHAw. 1995. Writing Ethnographic Fieldnotes. The University of Chicago Press, USA.

EMLEN, DJ \& LW OrING. 1977. Ecology, sexual selection, and the evolution of mating systems. Science, 197:215-223.

FRAKE, CO. 1969. The ethnographic study of cognitive systems. Pp. 28-41 en: Tyler, S (ed.). Cognitive anthropolog. Holt, Rinehart \& Winston, Nueva York.

FranKLIN, W. 1974. The social behaviour of the vicuña. Pp 477-487 en: Geist, V \& F Walther (eds.). The behaviour of ungulates and its relation to management. IUCN. Morges.

FRANKLIN, W. 1982. Biology, ecology and relationships to man of the South American Camelids. Vol. 6. Pp. 457-489 en: Marer, MA \& HH Genoways (eds.). Mammalian Biology in South America. University of Pittsburgh, Pittsburgh.

GONZÁLEZ, TY. 2001. Lo animal en la cosmovisión mexica o mesoamericana. Pp: 23-51 en: González, TY (de.). Animales y plantas en la cosmovisión mesoamericana. Plaza y Valdés, Instituto Nacional de Antropología e Historia y Sociedad Mexicana para las Religiones. México.

Guber, R. 2001. La Etnografía: Método, Campo y Reflexividad. Enciclopedia Latinoamericana de Sociocultura y Comunicación. Grupo Editorial Norma. Buenos Aires.

KaltenBORN, BP; T BJERKE; JW NYAHONGO \& DR WilLiams. 2006. Animal preferences and acceptability of wildlife management actions around Serengeti National Park,Tanzania. Biod. Conserv., 15:4633-4649.

KeLLERT, SR \& EO WILSON. 1993. The Biophilia Hypothesis. Washington, D.C. Island Press.

KELLERT, SR. 1996. The value of life: Biological diversity and human society. Island Press. Nueva York.

Koford, CD. 1957. The vicuña and the Puna. Ecological Monographs, 27:153-219.

LAWRENCE, E. 1993. The sacred bee, the filthy pig, and the bat out hell: Animal symbolism as cognitive biophilia. Pp. 301-341 en: Kellert, SR \& EO Wilson (eds.). The Biophilia Hypothesis. Island Press. Washington.

LENT, PC. 1974. mother-infant relationships in ungulates. Pp 14-55 en: Geist, V \& F Walther (eds.). The behavior of ungulates and its relation to management. International Union for the Conservation of Nature and Natural Resources. Morges, Suiza.

Leuthold, W. 1977. African Ungulates: A Comparative Review of their Ethology and Behavioral Ecology. Springer-Verlag. Nueva York.

Lévi-Strauss, C. 1962. La pensée sauvage. Librairie Plon. Paris.

MenARD, N. 1982. Quelques aspects de la sociologie de la vigogne lama vicugna. Terre Et La Vie-revue D'Ecologie Appliquee, 36:15-35.

MORCKRIN,MH;EL BENNETH \&DT LABRUNA. 2005. Wildlifefarming: a viable alternative to hunting in tropical forest? WCS Working paper 23. Wildlife Conservation Society, New York

Piaget, J. 1969. The child's conception of the worl. Littlefield, Adams. Totowa, NJ.

PIKE, K. 1954. Language in relation to a unified theory of humanbehavior. Summer Institute of Linguistics, Glendale.

Quinn, C; M Huby; H Kiwasila \& JC Lovett. 2003. Local perceptions of risk to livelihood in semi-arid Tanzania. J. Environ. Manage., 68:1111-1119.

Quiroga Mendiola, M \& G Ramisch. 2010. ¿Pastores o asalariados? Estrategias de vida en la continuidad y la coyuntura política en las altas montañas del noroeste de argentina. VIII Congreso Latinoamericano de Sociología Rural, Porto de Galinhas, Brasil.

Robinson, JG \& KH ReDFORD. 1997. Usos comerciales y de subsistencia de la vida silvestre en América Latina. Pp 23-42 en: Robinson, JG \& KH Redford (eds.). Uso y conservación de la vida silvestre neotropical. Fondo de cultura Económica. México.

Rojo, V; Y ARZAMENDIA \& B Vilá. 2012. Uso del hábitat por vicuñas (Vicugna vicugna) en un sistema agropastoril en Suripujio, Jujuy. Mast. Neot., 19:127-138.

SAntos Rodríguez, A. 2009. Metodología de la investigación etnozoológica. En: Madeiros Costa Neto, E; D Santos Fita \& M Vargas Clavijo (coord.). Manual de Etnozoología: Una guía teórico-práctica para investigar la interconexión del ser humano con los animales. Tundra Ediciones. España.

SMITH, K; CB BARRETT \& PW Box. 2001. Not necessarily in the same boat: Heterogeneous risk assessment among east African pastoralists. J. Develop. St., 37:51-30.

StURTEVANT, WC. 1968. Studies in ethnoscience. Pp. 475-500 en: Theoryin anthropology: A sourcebook. Manners, RA y D Kaplan (eds.). Routledge \& Kegan Paul, Londres.

Turbay, S. 2002. Aproximación a los estudios antropológicos sobre la relación entre el ser humano y los animales. Pp. 87-111 en: Ulloa, A (ed.). Rostros culturales de la fauna: las relaciones entre los humanos y los animales en el contexto colombiano. Instituto Colombiano de Antropología e Historia. ICANH.

URTON, G. (ed.). 1985. Animal myths and metaphors in South America. Univerity of Utha. Salt Lake City.

Vargas Clavijo, M. 2009. Patrimonio sociocultural: El mundo animal en las expresiones tradicionales de los pueblos. Pp. 118-144 en: Madeiros Costa Neto, E; D Santos Fita \& M Vargas Clavijo (coord.). Manual de Etnozoología:Una guía teórico-práctica para investigar la interconexión del ser humano con los animales. Tundra Ediciones. España.

VILA, BL \& MHCASSINI. 1993. Summer and autumn activity patterns in the vicuña. St. Neot. F. Environ., 28:251-258.

VILÁ, BL. 1992. Vicuñas (Vicugna vicugna) agonistic behaviour during the reproductive season. Pp. 475-782 en: Spitz, F; G Janeau G; G Gonzalez \& S Aulagnier (eds.). Ongules/Ungulates/91, Proceedings of the International Symposium. SFEPM y IRGM. Toulosse.

VILÁ. BL \& MH CASSINI. 1994. Time allocation during the reproductive season in the vicuña. Ethology, 97:226-235.

WAWRZYK, AC \& BL VILÁ. 2013. Dinámica del pastoreo en dos comunidades (Lagunillas del Farallón y Suripujio) de la puna de Jujuy, Argentina. Chungara: Revista de Antropología Chilena, 45:349-362.

WaWrZYK, AC; Y ARZAmendia; J Baldo \& H Lamas. 2009. Factibilidad para el manejo de vicuñas en silvestría en el noroeste de la puna jujeña, Argentina. En: V Congreso Mundial de Camélidos. Riobamba Ecuador. Asociación deLlamingueros del Ecuador "INTINAN" y la Diócesis de Riobamba. 


\section{INFORMACIÓN SUPLEMENTARIA}

\section{ANEXO 1: CUESTIONARIO GUÍA PARA LAS ENTREVISTAS EN PROFUNDIDAD}

¿Hay vicuñas en los lugares donde usted pastorea? ¿Cómo se llaman esos lugares?

¿Qué cantidad de vicuñas tienen los grupos que usted suele ver?

¿Cree que ahora hay más vicuñas que hace 20 años?

¿Sabe cómo se organizan las vicuñas? (en grupos, de cualquier forma, solas, etc.)

¿Qué tipo de organización es la que se ve con mayor frecuencia? (grupos familiares machos solteros, individuos solitarios)

¿Sabe cómo se conforman los grupos familiares?

¿Sabe cómo se conforman los grupos de solteros?

¿Sabe distinguir entre machos y hembras? ¿Cómo lo sabe?

¿Sabe qué comen las vicuñas?

¿Las vicuñas prefieren estar en algún lugar en particular?

¿A qué hora del día suele ver las vicuñas?

¿Por dónde las suele ver? (pasturas, agua, cerro)

¿Sabe dónde duermen las vicuñas? 